\title{
Modélisation du manteau neigeux à différentes échelles. Application au domaine des avalanches, de l'hydrologie et du climat
}

\author{
E. Brun, E. Martin \\ Météo-France/Centre National de Recherches Météorologiques/Centre d'Etudes de la Neige
}

\section{I — LES DÉBUTS DE LA MODÉLISATION DU MANTEAU NEIGEUX}

Le manteau neigeux joue un rôle important parmi les différents matériaux composant la surface terrestre car la neige présente des propriétés physiques tout à fait spécifiques. C'est particulièrement le cas pour ce qui concerne ses propriétés radiatives. Pour ces raisons, différentes branches des sciences de l'Univers se sont intéressées au manteau neigeux : hydrologie, climatologie, glaciologie, étude des risques naturels et écologie. Une meilleure connaissance des interactions entre l'atmosphère et la neige a focalisé le travail de nombreuses équipes de recherche. La compréhension des différents processus qui y participent a tout naturellement été synthétisée dans des modèles numériques qui s'attachent à simuler de la façon la plus fidèle possible l'évolution du manteau neigeux en fonction des conditions météorologiques qui la gouvernent. Outre la représentation des processus internes au manteau neigeux, une des principales difficultés est liée à la représentation de la très forte variabilité sur de faibles échelles d'espace qui caractérise le manteau neigeux.

C'est pour les besoins de l'hydrologie que les premiers modèles de neige ont été développés, de façon à calculer les effets de la fonte nivale sur l'écoulement des rivières en fonction des conditions météorologiques. Deux approches ont été adoptées :

- les méthodes par index qui relient la fonte du manteau neigeux à la température de l'air en utilisant des relations ajustées localement. Elles sont utilisées de façon opérationnelle sur de nombreux sites ou bassins versants ;

- les méthodes faisant appel à un bilan des échanges énergétiques entre l'atmosphère et la neige. Dans la suite de ce papier, ce sont celles-ci qui nous intéresseront car elles ont été à l'origine des premiers modèles numériques qui ont permis de calculer non seulement la fonte, mais aussi l'état interne du manteau neigeux.

Dans le domaine de la modélisation, des chercheurs français figurent parmi les précurseurs (Obled [1] et Navarre [2]) mais c'est le modèle d'Anderson [3] qui est reconnu par la communauté internationale des nivologues comme le premier modèle de neige basé sur les processus physiques.

Cette situation est sans doute due à l'effort de validation et de valorisation fait par Anderson dont le rapport publié par la NOAA en 1976 reste la référence sur les techniques de modélisation du manteau neigeux.

\section{LE MODÈLE DE NEIGE CROCUS}

C'est dans le milieu des années 80 que le CEN a de nouveau travaillé sur la modélisation du manteau neigeux pour les besoins de la Prévision du Risque d'Avalanches (PRA). Pour qu'un modèle de neige puisse intéresser cette activité, celui-ci doit être capable non seulement de simuler les profils de température et de teneur en eau liquide au sein du manteau neigeux, mais aussi le profil de densité et la stratigraphie. En effet, l'empilement de couches de neige, composées de cristaux de natures très différentes, est à la base de l'analyse de la stabilité du manteau neigeux.

Un travail en laboratoire a donc été mené pour quantifier expérimentalement les lois de métamorphose des différents types de neige soumis à des conditions couvrant une bonne part de celles qui peuvent être rencontrées dans les Alpes. Les travaux menés alors par Brunot [4] et Brun [5], portant respectivement sur la métamorphose de faible et moyen gradient et sur la métamorphose de la neige humide, ont complété les travaux antérieurs de Marbouty [6] sur les forts gradients de température. Un jeu de lois expérimentales a pu ainsi être introduit dans le modèle de neige Crocus pour simuler l'évolution cristallographique des différentes couches composant le manteau neigeux, de façon à représenter de manière acceptable les différents cas de métamorphose que l'on peut rencontrer dans les massifs montagneux de climat tempéré.

La simulation de la stratigraphie par un modèle de neige améliore très sensiblement le calcul du tassement et de l'albédo de la neige car ceux-ci peuvent alors dépendre du type et de la taille des grains composant les couches de neige. C'est là, le principal avantage de Crocus sur les autres modèles de neige. Les détails techniques de ce modèle sont décrits dans les articles [7] et [8].

\section{VALIDATION PONCTUELLE}

Le modèle Crocus a été testé sur le site instrumenté du Col de Porte (1 $320 \mathrm{~m}$, Massif de la Chartreuse). Le manteau neigeux a été simulé en fournissant au modèle les paramètres météorologiques mesurés sur ce site qui lui sont nécessaires comme données d'entrée, à savoir : tempéra- 
ture et humidité de l'air, vent, rayonnements solaire et atmosphérique incidents, précipitations liquide et solide. Les résultats ont été comparés aux mesures faites sur le manteau neigeux durant toute la saison $88 / 89$, aussi bien pour ce qui concerne la hauteur de neige, la température de surface, l'écoulement d'eau à la base que l'état interne du manteau neigeux [8]. L'hiver $88 / 89$ fut très propice au test car les conditions météorologiques rencontrées au $\mathrm{Col}$ de Porte ont couvert une large gamme d'événements, certains d'entre eux ayant même conduit à la formation de couches de gobelets, ce qui est relativement rare à $1320 \mathrm{~m}$ d'altitude. La qualité des résultats, illustrée sur les figures 1 et 2, a été jugée suffisante pour développer l'environnement nécessaire à une utilisation opérationnelle de Crocus pour les besoins de la PRA.

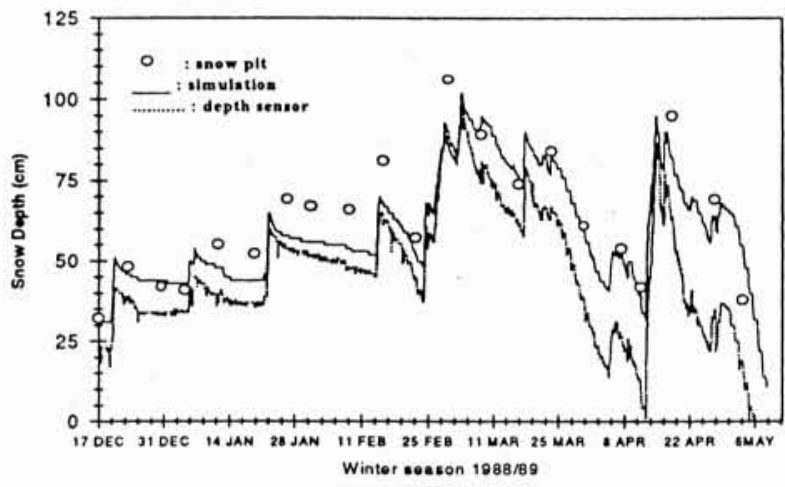

1. Comparaison entre hauteur de neige simulée par le modèle Crocus et observée sur le terrain.

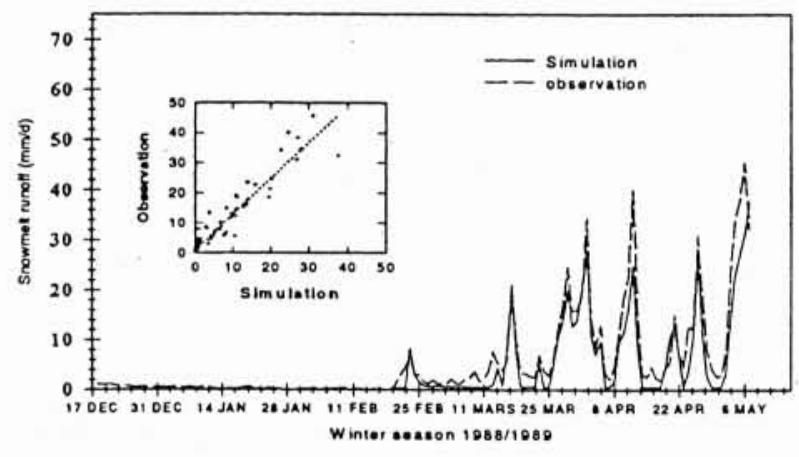

2. Comparaison entre écoulement d'eau à la base du manteau neigeux simulé par le modèle Crocus et observé sur le terrain.

\section{UTILISATION DE CROCUS L'ÉCHELLE RÉGIONALE}

\subsection{Modélisation du manteau neigeux sur les Alpes Françaises}

Pour utiliser un modèle qui calcule le bilan énergétique d'une surface de neige, il est évidemment nécessaire de lui fournir les variables météorologiques dont il a besoin en entrée. Celles-ci ne sont mesurées que sur de très rares sites. Pour simuler l'évolution du manteau neigeux sur un grand nombre de sites, une application météorologique a été spécialement développée. Celle-ci, nommée Safran, permet de calculer par massif, altitude et versant les paramètres météorologiques interagissant avec le manteau neigeux à partir de l'ensemble des données météorologiques disponibles opérationnellement : réseaux d'observations au sol (stations synoptiques, réseau nivométéorologique, stations automatiques), radio sondages entourant les Alpes, sorties de modèles météorologiques, images satellitaires et images radar [9]. Les flux radiatifs incidents sont calculés à partir de la nébulosité et du profil atmosphérique à l'aide d'un modèle de rayonnement [10]. Le gradient vertical de précipitation dépend, pour chaque massif, du type de temps selon une classification comprenant 10 étalons selon une méthode adaptée d'Aurhély [11]. Le schéma d'analyse de Safran est basé principalement sur les techniques d'interpolation optimale. Les Alpes Françaises sont découpées en 23 massifs considérés par hypothèse comme homogènes d'un point de vue climatologique et chaque massif est représenté par une série de pentes définies par leur altitude par pas de $300 \mathrm{~m}$ et par leur exposition (plat, N, E, SE, S, SW, W).

Sur chacune de ces pentes, Safran calcule les conditions météorologiques permettant de simuler l'évolution du manteau neigeux sur des pentes représentatives d'un massif, d'une altitude et d'une orientation. Evidemment, un tel système ne permet pas de simuler les phénomènes très locaux, tels que l'érosion ou l'accumulation de neige due au vent.

\subsection{Validation par simulation de l'enneigement quodi- dien}

La validation de la modélisation du manteau neigeux à l'échelle régionale à l'aide de Safran/Crocus a été conduite dans le cadre d'une étude sur la climatologie nivale des Alpes [12]. L'enneigement quotidien a été simulé sur la période 1981/1991 sur 37 sites répartis dans les Alpes pour lesquels on disposait de mesures de hauteur de neige entre 900 et $3000 \mathrm{~m}$ d'altitude. Pour chacun des sites, Safran ne connaissait que son massif, son altitude et son exposition, si bien qu'aucun ajustement n'a été fait pour prendre en compte la climatologie très locale des sites choisis. La comparaison entre les hauteurs de neige simulées et les observations quotidiennes fut bonne ou très bonne sur une trentaine de sites et mauvaise sur quatre sites. La figure 3 compare les résultats sur un des meilleurs sites. Deux causes sont à l'origine des quelques résultats décevants : - certains sites sont soumis à d'importants effets d'accumulation ou d'érosion de la neige par le vent qui ne sont pris en compte ni par Safran ni par Crocus,

- sur le seul site situé à $3000 \mathrm{~m}$, il y a une sousestimation des précipitations observable pour la plupart des épisodes. On ne peut cependant déterminer si ce défaut est dû aux caractéristiques locales de ce site ou s'il est dû à une sous-estimation systématique du gradient vertical des précipitations. L'enneigement moyen simulé sur 10 ans pour 4 décades sur les 37 sites a été comparé à celui observé sur le terrain, prouvant que la simulation du manteau neigeux à l'aide de Safran/Crocus est particulièrement adaptée au calcul de la climatologie nivale. De tels résultats ont permis des études systématiques de la sensibilité de 
l'enneigement à des variations de paramètres météorologiques ainsi qu'à des scénarios climatiques calculés par des modèles de climat [12]. Un modèle de neige basé sur la physique calculant le bilan énergétique neige/atmosphère tel que Crocus est particulièrement adapté car il n'utilise pas de relations statistiques reliant la fonte à un paramètre météorologique particulier et établies à partir d'observations. L'influence des différents paramètres sur l'enneigement peut être quantifiée avec une bonne fiabilité.

\subsection{Validation par simulation des débits d'une rivière alpine}

Les modèles hydrologiques utilisés pour simuler le débit d'une rivière fortement influencée par la neige calculent généralement la fonte de la neige à l'aide d'une méthode par index. Ces méthodes utilisent le plus souvent la température observée sur un ou plusieurs sites représentatifs pour déterminer la limite pluie-neige et le taux de fonte. La température de l'air n'étant pas le seul paramètre météorologique intervenant dans le bilan énergétique de la neige, son effet sur la fonte est déterminé par calibration sur le bassin versant de la rivière étudiée à partir de séries de débits passés.

Pour valider Safran/Crocus, notamment à haute altitude, les écoulements d'eau à la base du manteau neigeux calculés par ces deux modèles ont été utilisés pour forcer le modèle hydrologique ETH/HBV sur le bassin versant de la Romanche en amont du Chambon [13]. Les mesures de débit ont été fournies par le Service Ressources en Eau d'EDF. Le bassin a été subdivisé en parcelles caractérisées par leur altitude (bandes de $200 \mathrm{~m}$ ), leur exposition (N, S ou plat) et leur massif (Grandes-Rousses ou Oisans). Safran et Crocus ont été utilisés à partir des observations météorologiques disponibles, entre 1981 et 1991, pour simuler quotidiennement sur chacune des parcelles le manteau neigeux, l'écoulement d'eau à sa base ainsi que les précipitations liquides hors période d'enneigement. Ces données pondérées par la surface de chaque parcelle ont été utilisées pour forcer le modèle hydrologique préalablement calibré avec une représentation du manteau neigeux par une méthode par index. Les premiers résultats ont été surprenants : les débits liés au début de la fonte étaient bien simulés tandis qu'il apparaissait certaines années une forte surestimation du pic de débit printanier. Ces résultats étaient d'autant plus curieux que la comparaison des simulations avec l'enneigement, observé ces années-là en un point proche du bassin, montrait un comportement très réaliste de Safran/Crocus. Il est alors apparu que les problèmes venaient du fait que l'on appliquait la lame d'eau de fonte d'une parcelle à toute la surface de la parcelle lorsque son enneigement n'était pas nul. Or, une parcelle est définie par son massif, sa bande d'altitude et son exposition. Dans la réalité, une telle parcelle inclut des zones d'érosion (crêtes, rochers, croupes) et des zones d'accumulation (combes, pièges à neige), si bien qu'une partie de la parcelle est très vite déneigée dès le début de la fonte tandis qu'une autre reste enneigée même lorsque la neige a généralement disparu à l'altitude et sous l'exposition considérées. Appliquer la fonte à toute la surface surestime les débits en période de fonte très active. Pour corriger ce défaut, chaque parcelle a été subdivisée en 5 sous-parcelles :

- 1 parcelle occupant $30 \%$ de la surface et recevant $100 \%$ des précipitations moyennes sur la parcelle ;

- 2 parcelles occupant chacune $20 \%$ de la surface et recevant respectivement 50 et $150 \%$ des précipitations ;

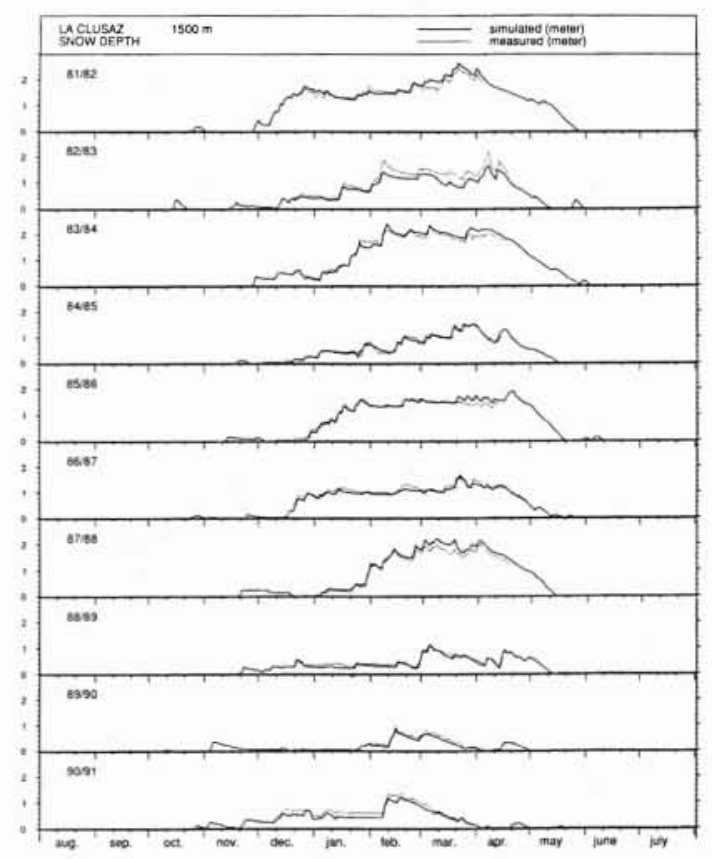

3. Comparaison entre l'enneigement quotidien mesuré à La Clusaz entre 1981 et 1991 et celui simulé par Safran/Crocus dans les Aravis, sur terrain plat à $1500 \mathrm{~m}$ d'altitude. 
- 2 parcelles occupant chacune $15 \%$ de la surface et recevant respectivement 25 et $175 \%$ des précipitations.

On peut ainsi représenter la diversité naturelle de l'enneigement due aux zones d'érosion et aux zones d'accumulation, de façon très simplifiée mais tout en conservant les précipitations totales.

Les résultats obtenus alors ont confirmé l'intérêt d'une telle approche, entraînant une nette amélioration les années présentant la surestimation du pic printanier sans détériorer les autres années. L'utilisation de Safran/Crocus pour forcer le modèle hydrologique ETH/HBV sans calibration propre à celle-ci, donne des résultats comparables aux résultats obtenus avec la version classique de ce modèle, voire légèrement améliorés (fig. 4).

Cette expérience a néanmoins prouvé qu'il était nécessaire de prendre en compte les effets du transport de neige par le vent, pour intégrer spatialement la série de résultats ponctuels calculés par Safran/Crocus.

\subsection{Modélisation du manteau neigeux des Alpes fran- çaises dans un contexte opérationnel}

La première mise en œuvre de Safran/Crocus dans un contexte opérationnel remonte aux Jeux Olympiques. Durant les Jeux, Météo-France a fourni une assistance au COJO pour les prévisions météorologiques et pour la prévision du risque d'avalanches. L'équipe chargée de la PRA a utilisé la modélisation du manteau neigeux dans les trois massifs où se déroulaient les Jeux (Vanoise, HauteTarentaise et Beaufortin), non seulement pour suivre en temps quasi réel l'évolution du manteau neigeux, mais aussi pour forcer le système expert MEPRA [14]. MEPRA analyse la stabilité du manteau neigeux associée à chacune des simulations faites par Crocus et synthétise cette information à destination des prévisionnistes. Le succès rencontré par Safran/Crocus/Mepra auprès de ses utilisateurs nous a conduit à étendre l'expérience aux autres massifs alpins. Depuis l'hiver 92/93, ces trois modèles sont émulés quotidiennement. Leurs résultats sont utilisés par le Centre Départemental de la Météorologie de Grenoble-SaintMartin d'Hères pour la PRA opérationnelle. Météo-France étudie actuellement l'extension de l'application aux autres centres météorologiques chargés de la PRA dans les Alpes, les Pyrénées et la Corse. Trois années d'utilisation de Safran/Crocus/Mepra ont montré que la qualité des trois modèles était tout à fait suffisante pour garantir la fiabilité de cet outil d'aide à la décision. Néanmoins, le découpage en altitude et versant n'est pas suffisamment fin pour représenter toute la variabilité naturelle du manteau neigeux. Il permet seulement de décrire l'état «typique » ou "moyen " du manteau neigeux ainsi simulé. Une étude financée par le Contrat de Plan Etat/Région a été engagée pour mieux représenter les effets du transport de neige par le vent sur l'évolution du manteau neigeux. Basée sur les observations faites sur le site du Col du Lac Blanc dans les Petites Rousses, cette étude devrait aboutir au couplage des modèles Safran/Crocus/Mepra, avec le modèle Elsa [15] qui devrait permettre de simuler la répartition des accumulations de neige en fonction du vent et de la topographie locale. Une fois validée, une telle approche pourrait offrir des perspectives intéressantes pour le suivi plus détaillé de certains sites.

\section{MODÉLISATION DU MANTEAU NEI- GEUX DANS UN MODÈLE MÉTÉORO- LOGIQUE}

Le manteau neigeux ne concerne pas les seules régions montagneuses. La plus grande partie des régions enneigées est relativement plate (Antarctique, Sibérie, Amérique du Nord). Dans ces régions, le manteau neigeux joue un rôle important sur les conditions météorologiques locales mais leur superficie est telle qu'il influence aussi le climat global de la terre. Celui-ci est simulé à l'aide de modèles de circulation générale (GCM) qui représentent le manteau neigeux de façon généralement simplifiée.

Dans le but de représenter de façon plus détaillée le manteau neigeux dans de tels modèles, nous avons introduit le modèle de neige Crocus dans le modèle météorologique global Arpège et dans sa version aire limitée Aladin. Arpège/IFS est un modèle spectral à maille variable développé conjointement par le Centre national de recherches météorologiques (Météo-France) et le Centre européen pour la prévision météorologique à moyen terme (Reading).

Le principe retenu pour un tel couplage consiste à faire évoluer les caractéristiques du manteau neigeux d'une maille à partir des flux d'énergie et de masse à l'interface sol/atmosphère calculé par Arpège. Les propriétés thermiques et radiatives du manteau neigeux ainsi simulé sont utilisées à leur tour par le modèle météorologique pour calculer les échanges sol/atmosphère au pas de temps suivant. Les premiers tests faits sur l'Europe de l'Ouest avec Aladin (maille horizontale d'environ $15 \mathrm{~km}$ ) ont montré des différences sensibles entre la version du modèle utilisant une paramétrisation simple de la neige et celle utilisant Crocus.

Des tests similaires ont été effectués avec la version climat d'Arpège en résolution T42. Bien qu'au stade actuel de développement seules les propriétés thermiques de la neige aient été modifiées (conduction et inertie), l'impact sur le climat des régions enneigées est important, comme l'illustre la figure 5. Il est apparu une augmentation du gradient vertical dans la couche limite de surface qui devient alors d'une grande stabilité thermique. Des tests sont en cours pour évaluer différentes prises en compte de cette forte stabilité dans les échanges turbulents.

L'introduction de Crocus dans un GCM est en cours de validation. D'ores et déjà, une telle approche offre des perspectives tout à fait intéressantes pour les études de l'influence du climat sur le pergélisol, sur l'hydrologie des régions enneigées et sur les échanges énergétiques et massiques à la surface des calottes glaciaires.

\section{VI $\square$ CONCLUSION}

La modélisation numérique s'est avérée une méthode efficace pour calculer les échanges d'énergie et de masse à l'interface neige/atmosphère et pour simuler l'évolution de l'état interne du manteau neigeux, y compris sa stratigraphie. L'extension spatiale de modélisations ponctuelles est possible mais son intégration nécessite de prendre des précautions, notamment pour la prise en compte des effets du transport de neige sur la répartition du manteau neigeux. 

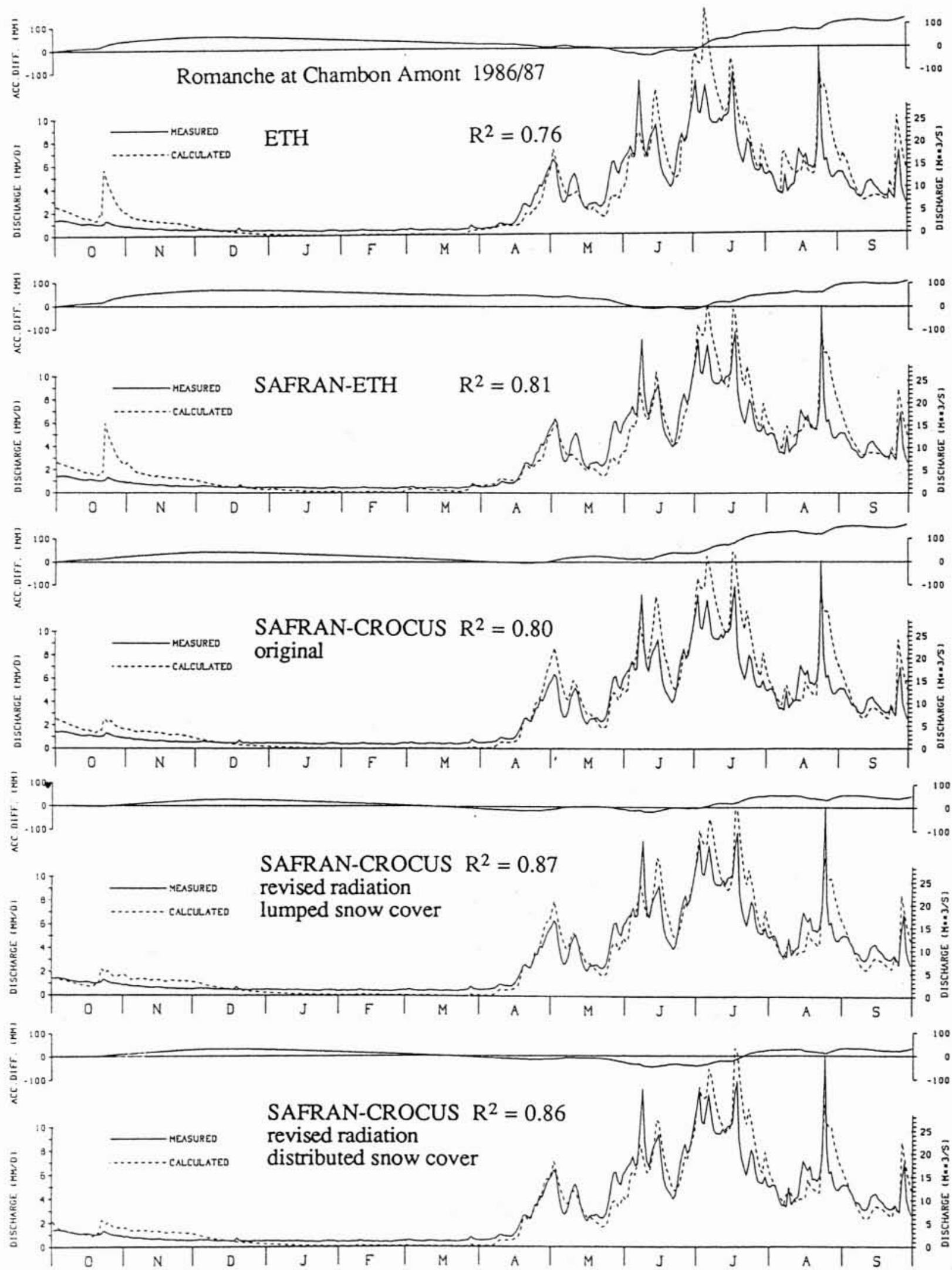

4. Comparaison entre les débits de la Romanche simulés et les débits observés (EDF/DTG) pour différentes versions de modèles (d'après Ludwig Braun). 
D'ores et déjà, la modélisation numérique du manteau neigeux peut être utilisée opérationnellement pour la prévision du risque d'avalanches et dans un contexte de recherche dans le domaine du climat et de l'hydrologie.

\section{Bibliographie}

[1] OBLED (1971). - Modèle mathématique de la fonte nivale. Thèse Université Scientifique et Médicale de Grenoble.

[2] NAvARre J.-P. (1975). - Modèle unidimensionnel d'évolution de la neige déposée. Modèle perce-neige. La Météorologie, 4 (3), 103-120.

[3] ANDERSON E. (1976). - A point energy and mass balance model for a snow cover. NOAA. Tech. Rep. NWS 19.

[4] BRUNOT G. (1986). - Etude quantitative de la métamorphose d'isothermie ou de faible gradient de la neige sèche. Note de travail de l'Ecole Nationale de la Météorologie.

[5] BRUN E. (1989). - Investigation on wet-snow metamorphism in respect of liquid water content. Ann. Glaciol., 13, 22-26.

[6] MARBoutr D. (1980). - An experimental study of temperature gradient metamorphism. J. Glaciol., 26 (94), $303-$ 312.

[7] Brun E., Martin E., Simon V., Gendre C. and Coléou C. (1989). - An energy and mass model of snow cover suitable for operational avalanche forecasting. J. Glaciol., 35 (121), 333-342.

[8] Brun E., David P., Sudul M. and Brunot G. (1992). A numerical model to simulate snow cover stratigraphy for operational avalanche forecasting. J. Glaciol., 38 (128), 13-22.

[9] Durand Y., Brun E., Mérindol L., Guypmarc'h G., LESAFFRE B. and MARTIN E. (1993). - A meteorological estimation of relevant parameters for snow models. Ann. Glaciol., 18, 65-71.

[10] GELEYN J.-F. and RITTER B. (1992). - A comprehensive radiation scheme for numerical weather prediction models with potential applications in climate simulations. Monthy Weather Review, 120 (2), 303-325.

[11] Bénichou P. and Le Breton O. (1987). - Prise en compte de la topographie pour la cartographie des champs pluviométriques statistiques. Météorologie, 7 (19), 23-34.

[12] Martin E., BRUn E. and Durand Y. (1994). - Sensitivity of the French Alps snow cover to the variation of climatic variables. Ann. Geophysicae, 12, 469-477.

[13] Braun L., Brun E., Durand Y., Martin E. and Tourasse P. (1994). - Simulation of discharge using different methods of meteorological data distribution, basin discretization and snow modelling. Nordic Hydrology 25, 129-144.

\section{Temperature at $2 \mathrm{~m}$ (Celsius) DJF 1989}

\section{ARPEGE/sans Crocus simulation reference (ne0)}

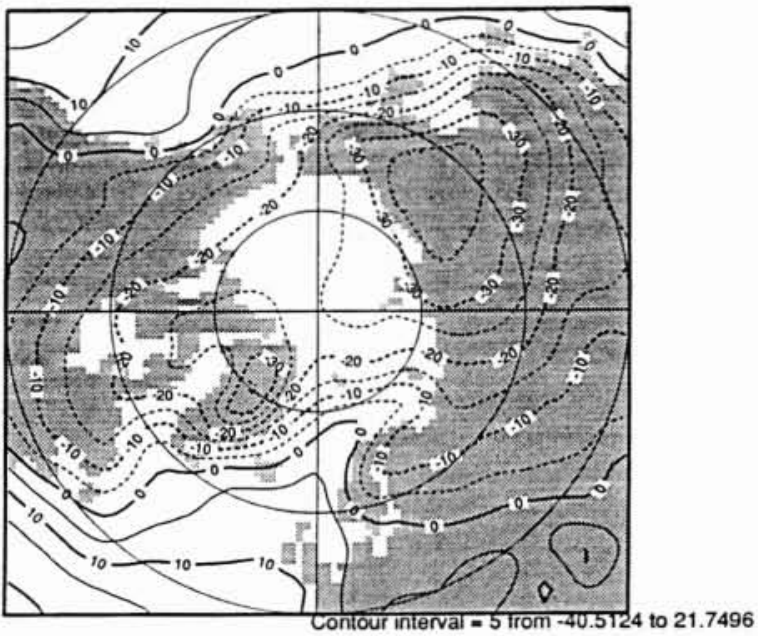

ARPEGE/Crocus simulation test (ts)

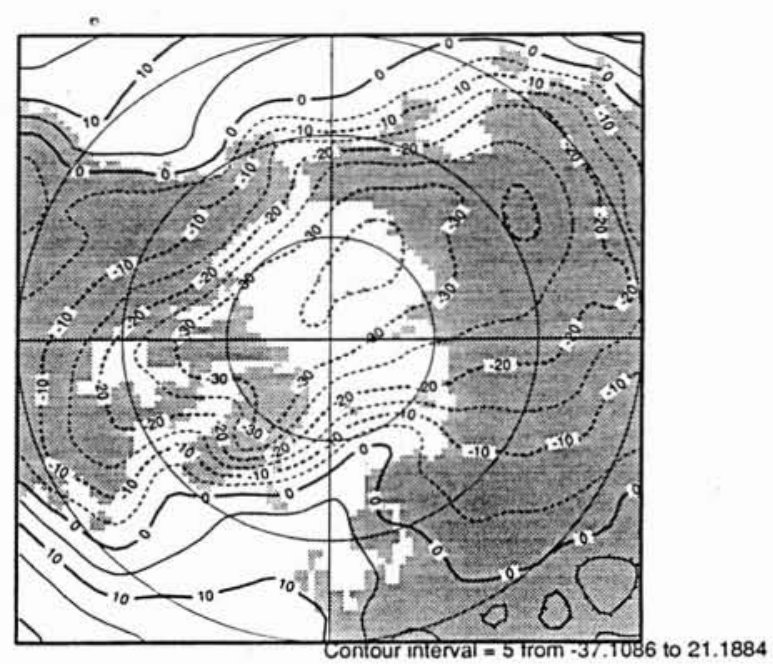

5. Comparaison entre les températures de l'air à $\mathbf{2} \mathbf{~ m}$ simulées pour la période décembre, janvier, février, avec la version climat d'Arpège et la version intégrant Crocus. Projection polaire de l'Hémisphère Nord.

[14] GIRAUD G. (1991). - MEPRA : Modèle expert d'aide à la prévision du risque d'avalanches. Symposium ANENA CISA-IKAR. Chamonix, 4-8 juin 91, 248-254.

[15] Buisson L. and CHARLIER C. (1993). - Avalanche modelling and integration of expert knowledge in the ELSA system. Ann. Glaciol., 18, 123-128. 